\title{
Macroion shielding and state-dependent pair potentials in colloidal suspensions
}

\author{
R Klein ${ }^{1}, \mathbf{H}$ H von Grünberg ${ }^{1}, \mathrm{C}$ Bechinger $^{1}$, M Brunner $^{1}$ and \\ V Lobaskin ${ }^{1,2}$ \\ ${ }^{1}$ Fakultät für Physik, Universität Konstanz, 78457 Konstanz, Germany \\ ${ }^{2}$ Soft Condensed Matter Group, Institute of Physics, University of Fribourg, CH-1700 Fribourg, \\ Switzerland
}

\begin{abstract}
We determine radial distribution functions for charge-stabilized colloidal suspensions for various volume fractions. By means of two independent inversion routines based on (i) the Ornstein-Zernike equation and (ii) the inverse Monte Carlo method, we extract effective colloid-colloid pair potentials from the distribution functions. Working at a fixed salinity, these potentials would not depend on the colloid density if the interactions between the colloids were purely pairwise additive. Our potentials however show a density dependence; agreement with the Yukawa form of DLVO (Derjaguin-Landau-VerweyOverbeek) theory is found only at low densities, but not at high densities. Such a state dependence of pair potentials reveals the density-dependent impact of many-body terms in the total interaction energy of the system. We also perform primitive model calculations, with parameters simulating our experiment, and find qualitatively similar deviations of the effective pair potentials from the Yukawa form, which thus confirms our experimental findings. Using results from a calculation of three-body interactions in colloidal suspensions, we are able to trace the observed density dependence of the pair potentials back to a shielding effect of the macroions. This interpretation is consistent with other experimental studies observing attractive parts in the pair potentials which were interpreted as 'like-charge attraction'. Our results, however, suggest that this attraction is a many-body effect.
\end{abstract}

\section{Introduction}

Macroscopic properties of colloidal suspensions [1,2] have their origin in the microscopic interactions between the constituents of the system. These interactions are fairly simple in suspensions of polymer-stabilized particles which behave to good approximation as hard spheres. The situation is, however, more complicated when the suspension is charge stabilized. 
It is then necessary to take account of the interactions between all charged species, which includes the highly charged colloids, the counterions and possibly the ions of added electrolyte. When the charged particles are described as charged hard spheres and the solvent as a dielectric continuum, one speaks of the primitive model [3]. The pair distribution functions (PDFs) of the colloidal particles are experimentally accessible, either through scattering experiments $[4,5]$ or by direct observation in real space. These PDFs can be related to pair potentials by liquid state integral theories. However, the pair potentials thus obtained describe the interactions between the constituents of an effective one-component model (OCM); as 'effective' potentials they are thus to be distinguished from the Coulomb potentials appearing in the primitive model of the original many-component system. The problem of how the direct Coulomb potentials can be related to the effective potentials obtainable from experiment is not a simple one and is, in fact, a subject of considerable recent interest $[2,6]$.

Within the framework of DLVO theory $[7,8]$, the effective pair interactions between the colloids in a charge-stabilized suspension are given by the Yukawa potentials. From several experimental observations $[9,10]$, there is evidence that this result needs refinement under certain circumstances, such as for very low salt concentrations which give rise to a rather wide spreading of counterions (thick double layers). Several effects not included in the DLVO theory have been considered, among them small-ion correlations and many-body interactions. The first of these is known to be particularly important for multivalent small ions. Restricting ourselves to the monovalent case where such effects are less important, we concentrate in this paper on the question whether effective potentials between macroions of other than twobody character may influence PDFs. We will demonstrate that provided that the answer to this question is positive, it is possible to obtain at least qualitative information about the importance of higher-order potentials from experiment by careful analysis of measured PDFs.

The problem of finding information about $N$-body $(N>2)$ potentials from the PDF is subtle and needs explanation. According to the McMillan-Mayer theory of mixtures the PDF between two macroions at $\boldsymbol{r}_{1}$ and $\boldsymbol{r}_{2}$ is given by the integral of the Boltzmann factor of the (many-macroion) potential of mean force, taken over all macroion positions except $\boldsymbol{r}_{1}$ and $\boldsymbol{r}_{2}$. The potential of mean force, on the other hand, is the sum of two-body potentials $v^{\text {eff }}\left(r_{i j}\right)$, threebody potentials $w^{\text {eff }}\left(\boldsymbol{r}_{1}, \boldsymbol{r}_{2}, \boldsymbol{r}_{3}\right)$, etc, which can in principle be derived from the interactions on the level of the primitive model. This approach of obtaining the PDF $g(r)$ has to be contrasted to the one usually used to interpret measured distribution functions in which a given $g(r)$ is related back to a pair potential $u^{\text {eff }}(r)$ using an Ornstein-Zernike (OZ) equation and an appropriate closure relation (CR). It is important to understand that since from the beginning only pairwise interactions are allowed for, this inversion of $g(r)$ results in an interpretation of the microstructure of the system in terms of pair potentials, even in cases where $N$-body $(N>2)$ interactions are non-negligible. Only in cases where $N$-body $(N>2)$ potentials of the McMillan-Mayer approach are absent or negligibly small will the pair potential $u^{\text {eff }}(r)$ of the $\mathrm{OZ}$ procedure coincide with the two-body potential $v^{\text {eff }}(r)$ of McMillan-Mayer theory. This is expected to be the case in highly diluted suspensions. Otherwise, the two potentials must differ and the difference between $u^{\text {eff }}(r)$ and $v^{\text {eff }}(r)$ expresses just the fact that there is an influence of three-body and $N$-body $(N>3)$ potentials on the $g(r)$. This we expect to happen at higher colloid densities.

Having said this, we can now describe the central idea of this paper: we first determine experimentally PDFs of a 2D colloidal system for varying colloid densities, using the same technique as in [11]. We, then, invert the $g(r)$ by means of the OZ equation, ending up with potentials $u^{\text {eff }}(r)$ for all measured colloid densities. According to what is said in the preceding paragraph, the set of curves $u^{\mathrm{eff}}(r)$ contains information about whether or not many-body contributions are important in our system: if many-body interactions are negligible, $u^{\text {eff }}(r)$ 
should be the same for all colloid densities; if not, we must observe a density effect in the potentials $u^{\text {eff }}(r)$. Indeed, we find that $u^{\text {eff }}(r)$ agrees well with the DLVO potential $v^{\text {eff }}(r)$ at low densities but deviates from $v^{\text {eff }}(r)$ at higher densities. This is therefore a direct experimental observation of many-body effects in colloidal suspensions.

The paper is organized as follows. Section 2 surveys the theoretical background, in particular what is meant by the two types of effective potential mentioned above. It furthermore reports some results for effective three-body potentials, relevant for the interpretation of the experiment described in section 3. The result of the experiment is a set of PDFs for widely differing densities. The inversion procedures used to obtain the effective pair potentials from $g(r)$ and the results of this inversion are presented in sections 4 and 5. Beside using two different inversion schemes we present in section 5 also MC simulation results for $g(r)$ starting from the primitive model. The results of this investigation are discussed in section 6 .

\section{Theoretical background}

\subsection{The Ornstein-Zernike equation in the one-component model}

Charge-stabilized suspensions are multicomponent fluids consisting of macroions, counterions, ions of added electrolyte and solvent molecules. A full statistical-mechanical description at this level is a rather complicated task, so various levels of coarse-graining have been developed. The lowest such level is the primitive model, which ignores the molecular nature of the solvent by replacing it by a dielectric continuum of relative dielectric constant $\varepsilon$. Here, the ionic species remain; they interact via short-range repulsive potentials which take care of finite diameters, and through Coulomb potentials $\beta v_{i j}(r)=Z_{i} Z_{j} l_{B} / r$. The subscripts $(i, j)$ refer to the charged species; $Z_{i}$ denotes the valency of species $i$ and $l_{B}=\beta e^{2} / \varepsilon$ with $\beta=1 / k_{B} T$ is the Bjerrum length. On this level, the suspension is a highly asymmetric electrolyte.

The microstructure and thermodynamic properties of the suspension can be expressed in terms of pair correlation functions, which obey coupled $\mathrm{OZ}$ equations:

$$
h_{i j}(r)=c_{i j}(r)+\sum_{k} \rho_{k} \int \mathrm{d} \boldsymbol{r}^{\prime} c_{i k}\left(\left|\boldsymbol{r}-\boldsymbol{r}^{\prime}\right|\right) h_{k j}\left(r^{\prime}\right) .
$$

Here, $h_{i j}$ and $c_{i j}$ denote total and direct correlation functions, respectively, and $\rho_{k}$ is the number density of species $k$. To make use of these equations, CRs are needed, which connect the correlation functions to the pair potentials $v_{i j}$. Their general form is

$$
g_{i j}=h_{i j}+1=\exp \left[-\beta v_{i j}+h_{i j}-c_{i j}+b_{i j}\right] .
$$

When the so-called bridge functions $b_{i j}$ are neglected, equation (2) is the hypernetted-chain (HNC) closure. Other approximate closures are obtained by various linearizations of the exponential (the Percus-Yevick (PY) and the mean-spherical approximation (MSA) CRs). Using the HNC closure, which is known to be fairly accurate for charged systems, the OZ equations have to be solved numerically. It turns out, however, that this scheme does not give convergent solutions for the case of highly asymmetric electrolytes such as colloidal suspensions, for which the valency $Z_{1}$ of the macroions is often as large as several hundreds or even thousands. The problem is easy to solve using the MSA closure, which puts $c_{i j}=-\beta v_{i j}$. For point-like particles it reproduces the Debye-Hückel theory, where

$$
h_{i j}=-\underset{i}{Z_{i} Z_{j} l_{B}} \mathrm{e}^{-\kappa_{D} r},
$$

and the screening parameter $\kappa_{D}$ is given by

$$
\kappa_{D}^{2}=4 \pi l_{B}\left(Z_{1}^{2} \rho_{1}+Z_{2}^{2} \rho_{2}\right) \equiv \kappa_{1}^{2}+\kappa_{2}^{2}
$$


for the case of no added electrolyte. The screening in equation (4) arises from the macroions (species 1$)$ and the counterions (species 2$)$. For monovalent counterions, $\kappa_{D}^{2}=4 \pi l_{B} Z_{1}\left(Z_{1}+\right.$ 1) $\rho_{1}$.

The next level of coarse-graining is to map the coupled OZ equations (1) onto an effective OCM, where only the coordinates of the macroions remain as statistical-mechanical degrees of freedom. The procedure is to rewrite the coupled system as one effective OZ equation for $h_{11}$. The result is of the form

$$
h_{11}(r)=c_{11}^{\text {eff }}(r)+\rho_{1} \int \mathrm{d} r^{\prime} c_{11}^{\text {eff }}\left(\left|r-r^{\prime}\right|\right) h_{11}\left(r^{\prime}\right),
$$

where $c_{11}^{\text {eff }}(r)$ is an effective direct correlation function. Although it is given formally in terms of the original $c_{i j}$ this is not of much help since they are not known (except in the MSA). To make use of equation (5) it is therefore necessary to connect $h_{11}$ and $c_{11}^{\text {eff }}$ to an effective pair potential $u^{\text {eff }}(r)$ between the constituents of the OCM which are now the macroions 'dressed' by their clouds of microions.

\subsection{Effective potentials}

One can describe the coarse-graining procedure from the primitive to the OCM in an alternative way, and this will provide us with information about the effective potential $u^{\text {eff }}(r)$. Following Beresford-Smith et al [14] one demands that the configurational integrals of the two models are the same. In the primitive model the configurational part of the Hamiltonian is $H\left(\left\{\boldsymbol{R}^{N_{1}}, \boldsymbol{r}^{N_{2}}\right\}\right)$; it depends on the positions $\left\{\boldsymbol{R}^{N_{1}}\right\}=\left\{\boldsymbol{R}_{1}, \ldots, \boldsymbol{R}_{N_{1}}\right\}$ of $N_{1}$ macroions and on the positions $\left\{\boldsymbol{r}^{N_{2}}\right\}$ of $N_{2}$ small ions. The condition of identical configurational integrals of the two models provides an average over all configurations of small ions and an expression for the $N_{1}$-body potential of mean force $H^{\text {eff }}\left(\left\{\boldsymbol{R}^{N_{1}}\right\}\right)$ of a fixed configuration of the macroions. This procedure guarantees that the thermodynamic properties of the OCM are the same as for the primitive model. The result of this approach is of the form

$$
H^{\text {eff }}\left(\left\{\boldsymbol{R}^{N_{1}}\right\}\right)=F+W\left(\left\{\boldsymbol{R}^{N_{1}}\right\}\right) .
$$

The quantity $F$ is independent of the configuration $\left\{\boldsymbol{R}^{N_{1}}\right\}$; it consists of the ideal-gas free energy of the small ions and so-called volume terms, which arise from the interactions and correlations among the small ions and the interactions of a single macroion with the small ions. Whereas this contribution is important for the thermodynamic behaviour of the suspension [15], it is not relevant for structural properties. The latter are determined from $W\left(\left\{\boldsymbol{R}^{N_{1}}\right\}\right)$, which can be presented as a sum of two-body, three-body etc state-dependent potentials:

$$
W\left(\left\{\boldsymbol{R}^{N_{1}}\right\}\right)=\sum_{i<j} v^{\mathrm{eff}}\left(\boldsymbol{R}_{i}, \boldsymbol{R}_{j}\right)+\sum_{i<j<k} w^{\mathrm{eff}}\left(\boldsymbol{R}_{i}, \boldsymbol{R}_{j}, \boldsymbol{R}_{k}\right)+\cdots
$$

Within Debye-Hückel theory only the two-body contributions remain [14]:

$$
\beta v^{\text {eff }}(r)=\begin{array}{cc}
Z_{1}^{2} l_{B} & \mathrm{e}^{-\kappa_{2}(r-2 a)} \\
\left(1+\kappa_{2} a\right)^{2} & r
\end{array}
$$

where the screening arises from the small ions only, and where $a$ denotes the macroion radius. This potential has the Yukawa form; it is also obtained if one calculates the total free energy of a system consisting of two colloids, using the solution to the linearized Poisson-Boltzmann (PB) equation [12]. Until now, we have considered only counterions (see the definition of $\kappa_{2}$ in equation (4)). If in addition a monovalent salt is added to the system, then the screening constant in equation (8) becomes

$$
\kappa_{2}^{2}=4 \pi \lambda_{B}\left(Z \rho+2 n_{s}\right)
$$


with $\rho=\rho_{1}$ and $Z=Z_{1}$ for the colloid density and charge, respectively. If the system is coupled to a salt reservoir (which is usually the case), then one has to distinguish between $n_{s}$, the salt concentration inside the suspension, and the salt concentration $c_{s}$ of the salt reservoir. In concentrated systems, showing a strong Donnan effect, $n_{s}$ is much smaller than $c_{s}$. On the other hand, if $Z \rho<n_{s}$, there is no Donnan effect [13] and $n_{s}$ becomes equal to $c_{s}$, so $\kappa_{2}$ then reduces to a value which is independent of the density of macroions:

$$
\kappa_{2}^{2}=8 \pi \lambda_{B} c_{s}
$$

For highly charged objects, for which the electrostatic energy of a microion in the vicinity of a macroion surface becomes much larger than $k_{B} T$, linearization of the PB equation is inadequate. However, at large separations of two macroions the potential has still the Yukawa form, equation (8), but with a renormalized charge, $Z_{1}^{\text {eff }}$ [16]. In systems where the screening constant is given by equation (10) (which we will see to be the case in our experiment), the individual terms in the sum of equation (7) are density independent.

Concerning the three-body potentials of mean force in equation (7) there are several results from simulations $[17,18]$, which show that for certain parameters chosen in these studies, the potentials can be attractive. The conventional procedure within the OCM has been to use the DLVO potential equation (8) in a CR for the OCM OZ equation, equation (5), and to neglect the higher-order terms completely. In such studies, one solves the OZ equation for a given CR in order to obtain the total correlation function, describing the microstructure of the suspension.

If precise experimental data for the pair correlation function $g(r)$ of macroions are available, one can also proceed in the opposite direction: the $\mathrm{OZ}$ equation in combination with the CRs can then be used to come from the measured $g(r)$ to the pair potential. This is the approach that we choose in this paper. As already pointed out in the introduction, this standard inversion procedure enforces an answer in which all interactions in the system are given in terms of pairwise interactions. When three-body and higher-order terms in the potential of mean force cannot be omitted, the approach of going from a measured $g(r)$ via the CR to a pair potential results in potentials $u^{\text {eff }}(r)$, which must also be called 'effective', but now in a sense that is different from how it is used in the McMillan theory. 'Effective' now refers to the fact that $u^{\text {eff }}(r)$ also contains to a certain (unknown) degree of contributions of higher-order interaction terms between the colloids ( $w^{\text {eff }}$ etc in equation (7)). In some sense the higherorder many-body potentials are absorbed into the pair potentials. Therefore it is clear that the $u^{\text {eff }}(r)$ thus obtained differs from the real pair potential $v^{\text {eff }}(r)$ in equation (7). With this new pair potential $u^{\text {eff }}(r)$, one can try to approximate the $N_{1}$-body potential of mean force in equation (7):

$$
W\left(\left\{\boldsymbol{R}^{N_{1}}\right\}\right) \approx \sum_{i<j} u^{\mathrm{eff}}\left(R_{i j}\right) .
$$

The main objective of this study is to investigate whether $u^{\text {eff }}(r)$ for highly charged colloids is of DLVO type, but with renormalized parameters, or whether it has a different functional form. It is clear that at low colloid density, when many-body interactions in equation (7) can be safely neglected, we should find that $u^{\text {eff }}(r)=v^{\text {eff }}(r)$, i.e. $u^{\text {eff }}$ has the Yukawa form. At higher densities, however, the higher-order terms in equation (7) become important, something which one should be able to identify by a difference between $u^{\text {eff }}(r)$ and $v^{\text {eff }}(r)$, which grows larger with increasing colloid density. In other words, we here study the functional form of $u^{\text {eff }}(r)$ for various systems of different colloid densities, and aim at identifying many-body interactions by finding density-dependent deviations of $u^{\text {eff }}(r)$ from the expected Yukawa form. 


\subsection{Estimates of the effective three-body potential}

Assuming that the difference between the DLVO-like potential $v^{\text {eff }}(r)$ and the effective pair potential $u^{\text {eff }}(r)$ arises mainly from the three-body terms in equation (7), we will try to determine the latter at least approximately and for a few typical arrangements of three particles. To this end, we have considered the grand potential $\Omega\left(\left\{\boldsymbol{R}^{N_{1}}\right)\right\}$ for the microions in the field created by $N_{1}$ macroions in a chosen fixed configuration $\left\{\boldsymbol{R}^{N_{1}}\right\}$. The microions are assumed to be in contact with a reservoir, which corresponds to the experimental situation as will be described later, so the description in terms of the grand canonical ensemble is appropriate. The grand potential can be calculated for the configuration $\left\{\boldsymbol{R}^{N_{1}}\right\}$ once the (normalized) electrostatic potential $\Phi(\boldsymbol{r})$ and therefore the distributions of positive and negative small ions $\rho_{ \pm}(\boldsymbol{r})=c_{s} \mathrm{e}^{\mp \Phi(r)}$ are known. The potential $\Phi(\boldsymbol{r})$ is the solution to the non-linear PB equation

$$
\nabla^{2} \Phi(\boldsymbol{r})=\kappa^{2} \sinh \Phi(\boldsymbol{r})
$$

in the region outside the macroions with constant-charge boundary conditions on the surfaces of the macroions. The screening constant is given by equation (10). Through the boundary conditions, the $\Phi(\boldsymbol{r})$ and therefore $\Omega\left(\left\{\boldsymbol{R}^{N_{1}}\right\}\right)$ depend on the positions of the macroions.

When there is only one macroion present $\left(N_{1}=1\right)$ the grand potential is just the selfenergy of the ion cloud surrounding the colloidal particle, $\Omega_{1}$, which is independent of the position of the macroion for a translation-invariant system. For two particles $\left(N_{1}=2\right)$, which are far away from each other, the grand potential is $\Omega_{2}=2 \Omega_{1}$. But when their separation $R_{12}$ is reduced, we have $\Omega_{2}\left(R_{12}\right)=2 \Omega_{1}+\Omega^{(2)}\left(R_{12}\right)$, where $\Omega^{(2)}\left(R_{12}\right)$ is the pair interaction potential. Having three particles in the configuration $\left\{\boldsymbol{R}_{1}, \boldsymbol{R}_{2}, \boldsymbol{R}_{3}\right\}$, the grand potential is

$\Omega_{3}\left(\boldsymbol{R}_{1}, \boldsymbol{R}_{2}, \boldsymbol{R}_{3}\right)=3 \Omega_{1}+\Omega^{(2)}\left(R_{12}\right)+\Omega^{(2)}\left(R_{13}\right)+\Omega^{(2)}\left(R_{23}\right)+\Omega^{(3)}\left(\boldsymbol{R}_{1}, \boldsymbol{R}_{2}, \boldsymbol{R}_{3}\right)$

where the pair potentials are taken from the previous step where $N_{1}=2$ and where the last term on the right-hand side, $\Omega^{(3)}\left(\boldsymbol{R}_{1}, \boldsymbol{R}_{2}, \boldsymbol{R}_{3}\right)$, is the three-particle interaction potential. Note the difference between $\Omega^{(3)}$ and $\Omega_{3}$, the first quantity being the three-body potential, the second the total grand potential for three particles in the system. To determine $\Omega^{(3)}\left(\boldsymbol{R}_{1}, \boldsymbol{R}_{2}, \boldsymbol{R}_{3}\right)=$ $\Omega^{(3)}\left(R_{12}, R_{13}, R_{23}\right)$, where $R_{i j}=\left|\boldsymbol{R}_{i}-\boldsymbol{R}_{j}\right|$, from equation (13) it is necessary to solve the PB equation (12) with the boundary condition for one particle to obtain $\Omega_{1}$, then to solve it for pairs of particles at separations $R_{i j}$ to obtain the three terms $\Omega^{(2)}\left(R_{i j}\right)$ and finally to solve the PB equation for three particles to obtain $\Omega_{3}\left(\boldsymbol{R}_{1}, \boldsymbol{R}_{2}, \boldsymbol{R}_{3}\right)$. Equation (13) can then be used to find the three-particle potential $\Omega^{(3)}\left(R_{12}, R_{13}, R_{23}\right)$. This procedure has recently been realized for certain sets of macroion positions [19].

For the present discussion, it suffices to consider the simplest of such macroion configurations, which is when their centres are on a straight line. Keeping the distance $R_{12}$ constant, $R_{12}=c_{12}$ and varying $R_{23}$, where particle 2 is between particles 1 and $3, \Omega^{(3)}$ and $\Omega_{3}$ both vary with $R_{23}$. Disregarding three-particle interactions, the grand potential of the three-particle system is given by equation (13) without the last term. The full calculation shows however that the three-particle contribution $\Omega^{(3)}\left(R_{23}\right)$ (i.e. $\Omega^{(3)}\left(R_{12}=c_{12}, R_{13}=\right.$ $\left.c_{12}+R_{23}, R_{23}\right)$ ) is negative and that it nearly cancels the positive pair contribution $\Omega^{(2)}\left(R_{13}\right)$ arising from the outer two particles [19]. This result shows that the DLVO-type repulsive interaction between a pair of macroions is strongly reduced or even cancelled when another macroion lies in between; macroion 2 strongly screens the interaction between 1 and 3 . Generalizing this finding to a suspension of many macroions it can be expected that the effects of three-particle interactions will modify the pair potential at distances larger than the mean interparticle separation $D$. Our expectation as to the difference between the potentials $v^{\text {eff }}(r)$ and $u^{\text {eff }}(r)$ is therefore that $u^{\text {eff }}(r)$ has the DLVO functional form for $r<D$, but that it will decay to zero much faster than $v^{\text {eff }}(r)$ when $r>D$. This crossover is density dependent. 


\section{An experiment to measure state-dependent potentials}

The experiments were performed using an aqueous suspension of highly charged $\left(9.7 \mu \mathrm{Ccm}^{-2}\right)$ surfactant-free, sulphate-terminated polystyrene (PS) particles with $3 \mu \mathrm{m}$ diameter and a polydispersity below 4\% (Interfacial Dynamics Corporation). As the sample cell we employed a cuvette with two optical flats of fused silica at a separation of $0.5 \mathrm{~mm}$. The cell was connected to a closed deionization circuit which additionally consisted of an electrical conductivity meter, a vessel of ion exchange resin, a reservoir basin and a peristaltic pump [20]. Before each measurement, the suspension was pumped through the ion exchanger to reduce the salt concentration and we obtained typical ionic conductivities below $0.07 \mu \mathrm{S} \mathrm{cm}^{-1}$. Afterwards the sample cell was mechanically disconnected from the circuit in order to guarantee stable and reproducible ionic conditions during the measurements. In order to confine our system to $2 \mathrm{D}$, we directed the beam of a frequency-doubled $\mathrm{Nd}$ : $\mathrm{YVO}_{4}$ laser with an intensity of $400 \mathrm{~mW}$ from above into the sample cell (figure 1). The vertical light pressure of the beam forces the negatively charged particles to the also negatively charged glass surface of the sample cell and thus reduces vertical particle fluctuations. The fluctuations were determined by total internal reflection microscopy to be less than $100 \mathrm{~nm}$ [21]. Since this corresponds to less than $4 \%$ of the particle diameter, the system can be regarded as 2D to good approximation. We have not observed any changes in the measured PDFs upon variation of the laser intensity of the $\mathrm{Nd}: \mathrm{YVO}_{4}$ laser. Therefore we can rule out light-induced effects in the pair interaction of particles such as optical binding [22]. The construction of our cell is such that the volume per colloidal particle is particularly large. This implies that the number of counterions in the volume is expected to be low compared to the total number of salt ions, regardless the fact that we are working at low-salt conditions. Therefore, the appropriate screening constant in our experiment is equation (10). Our experimental set-up is thus designed to suppress the trivial density dependence of the pair potential arising from the density dependence of the screening constant.

The sample was illuminated with white light through a dichroic mirror from above, to image the particles with a long-distance microscope objective (Zeiss, Achroplan $20 \times, 0.4$ ) onto a CCD camera chip (Teli, CS 8310), the latter being connected to a computer. The intense laser light was blocked below the sample cell with an optical absorber to protect the CCD camera from direct exposure to the high laser intensity. The particle centre positions were monitored with a lateral resolution of about $200 \mathrm{~nm}$ using an imaging processing system. To ensure that subsequent pictures were statistically independent from each other, only one picture per second was taken. In the case of low particle densities, up to 20000 pictures were recorded.

In order to study density-dependent effects in the effective pair potential, the particle density was adjusted by means of a scanned optical tweezer which acted as a boundary box for the 2D colloidal suspension investigated. The beam of an $\operatorname{argon-ion}$ laser $(\lambda=488 \mathrm{~nm}$, $P=200 \mathrm{~mW}$ ) was reflected from a 2D galvanostatically driven mirror and focused with a spot size of about $2 \mu \mathrm{m}$ onto the sample plane. The mirror position was controlled by a computer in such a way that the focus scanned a square with about $300 \mu \mathrm{m}$ side length. Because the repetition rate of the pattern was of the order of $50 \mathrm{~Hz}$, no net drift of the particles along the contour of the scanned laser focus is observed and the optical trap can be considered to be static [25] with a measured potential depth of $30 k_{B} T$ perpendicular to the contour of the square. Accordingly, the particles ordered in a $1 \mathrm{D}$ dense packing along the contour of the laser trap and thus served-due to their electrostatic interaction-as a corral for the particles inside the boundary box. Variation of the box size thus yields a convenient way to adjust the particle density inside the box [11]. In addition to the very precise and reproducible control 
of the particle density, this method has the advantage that one can perform measurements at different particle densities of an identical set of particles.

To check whether the confinement by the charged glass plate has any effect on the pair potentials, we computed the pair interaction between two colloidal spheres in front of a charged wall from the solution of the full PB equation, equation (12), for the set of parameters realized in our experiment $\left(a=1500 \mathrm{~nm}, Z^{\text {eff }}=25000, \kappa^{-1}=250 \mathrm{~nm}\right)$, and found no significant confinement effect. So, the potentials for unconfined systems, discussed in the previous section, should describe well the interaction in our confined geometry also.

\section{Results-inversion using the Ornstein-Zernike equation}

Pictures of the suspension such as that in figure (2) are subsequently analysed by calculating PDFs. In each picture we choose only a fraction of colloidal particles from the central region of the frame, each of which is then taken as a reference particle for the determination of $g_{N}(r)$. These reference particles must have a certain distance from the border of the system, because we are interested in the bulk PDF, unaffected by the symmetry break that is induced by the borderline. Here $N$ is the number of particles whose centres are located within the circle of radius $R_{\max }$ around the chosen reference particle; $\rho_{N}=N / \pi R_{\max }^{2}$ is then the density (number of particles per unit area). The slot size of the histogram needed to determine $g_{N}(r)$ is chosen in consistency with the optical resolution of our camera. Since the exact number of particles within $R_{\max }$ will vary from reference particle to reference particle and from frame to frame, $N$ (and thus $\rho_{N}$ and $g_{N}(r)$ ) depends implicitly on the frame and the reference particle. For a specific reference particle and frame, $2 \pi \rho_{N} \int_{0}^{R_{\max }} g_{N}(r) r \mathrm{~d} r=N-1$. The quantity $g(r)$ is now obtained from averaging these $g_{N}(r)$ over all frames and reference particles, $\rho^{2} g(r)=\left\langle\rho_{N}^{2} g_{N}(r)\right\rangle$ with the mean density $\rho=\left\langle\rho_{N}\right\rangle$. By construction,

so

$$
\rho^{2} 2 \pi^{2} R_{\max }^{2} \int_{0}^{R_{\max }} g(r) r \mathrm{~d} r=\left\langle N^{2}\right\rangle-\langle N\rangle
$$

$$
1+\rho \hat{h}(0)=\begin{gathered}
\left\langle\Delta N^{2}\right\rangle \\
\langle N\rangle
\end{gathered}
$$

with $\Delta N=N-\langle N\rangle$ and

$$
\hat{h}(0)=2 \pi \int_{0}^{R_{\max }}(g(r)-1) r \mathrm{~d} r .
$$

Figure (3) shows the PDFs (symbols) thus determined from the experimental data, for six different values of $\rho \sigma^{2}$ between $\rho \sigma^{2}=0.04$ and $\rho \sigma=0.19$, the latter value corresponding almost to the phase transition point where the suspension spontaneously crystallizes.

The measured $g(r)$ provides us with information about the total correlation, i.e. about $h(r)=g(r)-1$. To obtain the direct correlation function, we first have to deconvolute the $c(r)$ contained in the function $g(r)$, using the OCM OZ equation, equation (5). The determination of the effective potentials from $g(r)$ proceeds in a way described for instance in [23]. Specifically, our way of determining $c(r)$ is to first fast Fourier transform (FFT) the $h(r)$, thus obtaining $\hat{h}(q)=\int \mathrm{d} \boldsymbol{r} h(r) \mathrm{e}^{-\mathrm{i} q r}$. This is related to the Fourier transform of $c(r)$ via

$$
\hat{c}(q)=\begin{gathered}
\hat{h}(q) \\
1+\rho \hat{h}(q)
\end{gathered} .
$$

With a FFT back to direct space, we arrive at $c(r) . c(r)$ can now be related to the pair potentials by the CRs. As already pointed out above, all CRs produce correct pair potentials only for 


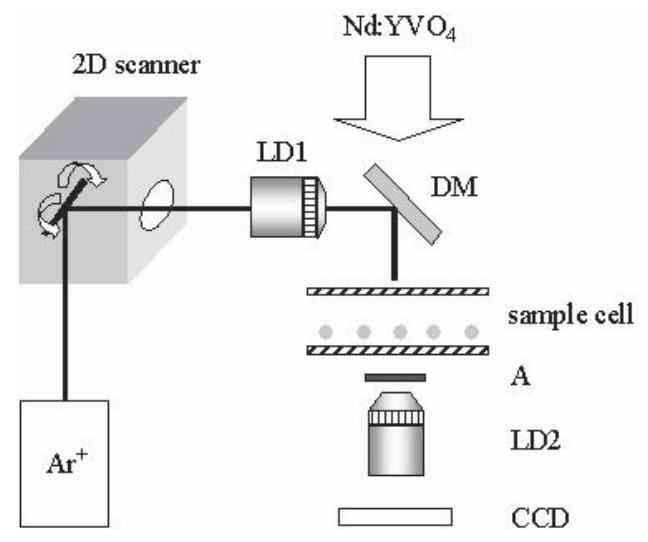

Figure 1. A schematic representation of the experimental set-up. A Nd:YVO laser forces the colloidal particles due to vertical light to form a $2 \mathrm{D}$ system. The particle density is adjusted by means of an $\mathrm{Ar}^{+}$laser which is deflected from a 2D galvanostatic scanner and directed with a microscope objective (LD1) and a dichroic mirror (DM) into the sample cell. Imaging of the particles is performed by a second microscope objective (LD2) and a CCD camera. To protect the camera from the high-intensity laser light, an optical absorber (A) is inserted underneath the sample cell.

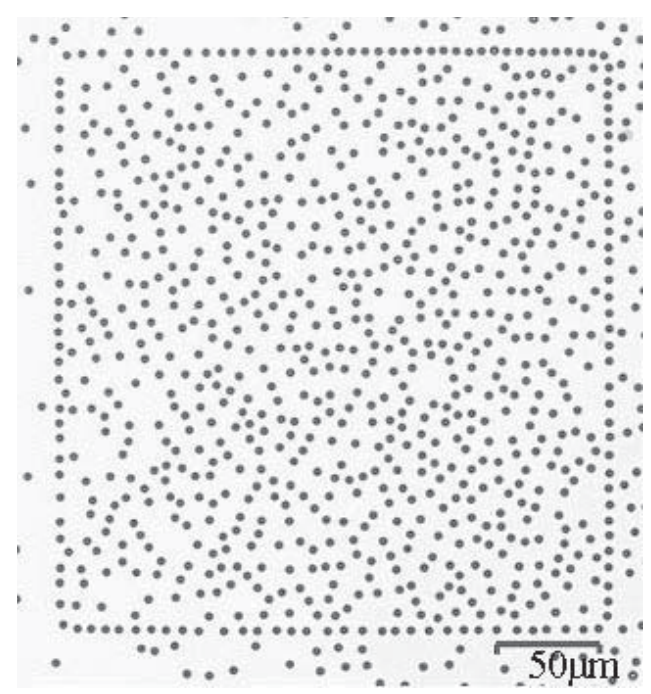

Figure 2. An optical image of the $2 \mathrm{D}$ colloidal suspension. The system under investigation is confined by an one-dimensional array of colloidal particles that are held fixed by light forces. The size of this confining box can be changed, thus enabling us to change the particle density of the 2D colloidal system in a controlled way.

low densities; they are bound to fail at higher colloid densities. Before using this inversion procedure, we therefore have to test the range over which the CRs produce reliable results.

This can be done with the help of MC simulation data. For that purpose, we first simulated a 2D colloidal system using a pair potential $\beta u^{\text {eff }}(r)=A \sigma \exp (-\kappa r) / r$, with $A=2.5 \times 10^{5}$ and $\kappa \sigma=5.5$. The resulting $g(r)$ was inverted via the OZ equation, in the way described above, to extract the pair potentials from the $g(r)$ which could then be compared to the potential actually used in the simulation. Ideally, both potentials should be identical. Figure 4 shows that for low 


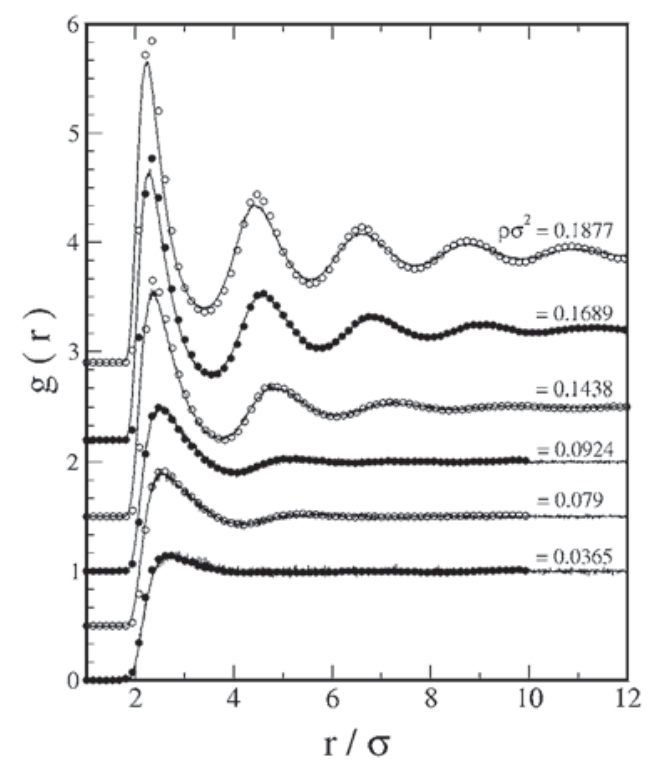

Figure 3. PDFs for various colloid densities $\rho \sigma^{2} . \sigma=2 a$ denotes the particle diameter. The variation of the density (for the same set of colloidal particles!) was realized by changing the box size of the 2D colloidal system inside the rectangular box in figure 2. Symbols: for the experimental data; solid curves: for MC data.

densities this is indeed the case. We compare the true potential with the potentials calculated using the HNC, PY and MSA CRs (in the MSA, $\beta u^{\text {eff }}(r)$ is given just by the negative of $c(r)$ at larger distances), and find almost perfect agreement for the HNC- and PY-based potentials if $\rho \sigma^{2}=0.036$ (figure 4(a)), and a fair agreement at high density, $\rho \sigma^{2}=0.14$ (figure 4(b)). It is obvious from a comparison of figures $4(a)$ and (b) that the difference between all four potentials increases with increasing $\rho$, which indicates that the error of the potential produced by the inversion method itself increases with the density. Luckily, it is found that at higher densities the true pair potential is bracketed by the HNC- and PY-based potentials, just like for 3D suspensions [1]. This offers a convenient way to keep control of the error produced by the inversion procedure, by considering the HNC-based potential as the upper bound and the PY-based potential as the lower bound of the true potential. Multiplying the potentials by $r$ and plotting them logarithmically, one expects straight lines for potentials that have the Yukawa form. Figure 4(c) demonstrates that due to the CRs the potentials resulting from the inversion routine do indeed show some deviations from the Yukawa form of the true potential (straight solid line).

Having thus checked for the error produced by our inversion procedure, we can now analyse the measured PDFs of figure 3. Figure 5 shows the potentials obtained from the inversion procedure using the HNC CR (solid curve) and the PY CR (dashed-dotted curve). Again, all potentials are multiplied by the radial distance and plotted logarithmically, to facilitate comparison with Yukawa potentials. Let us first consider the plots for the three lowest densities, (a)-(c) in figure 5. We here do indeed obtain potentials of almost perfect Yukawa form, just as standard DLVO theory predicts. We have fitted the $\rho \sigma^{2}=0.0924 \mathrm{HNC}$ curve to a function $\beta u^{\text {eff }}(r)=A \sigma \exp (-\kappa r) / r$, obtaining $A=2.5 \times 10^{5}$ and $\kappa \sigma=5.5\left(\kappa^{-1}=545 \mathrm{~nm}\right)$. This Yukawa potential is plotted as a reference potential in all five plots of figure 5 (thick dashed curves). Using $A=2.5 \times 10^{5}$ and $\kappa \sigma=5.5$ in a Yukawa interaction potential we performed 

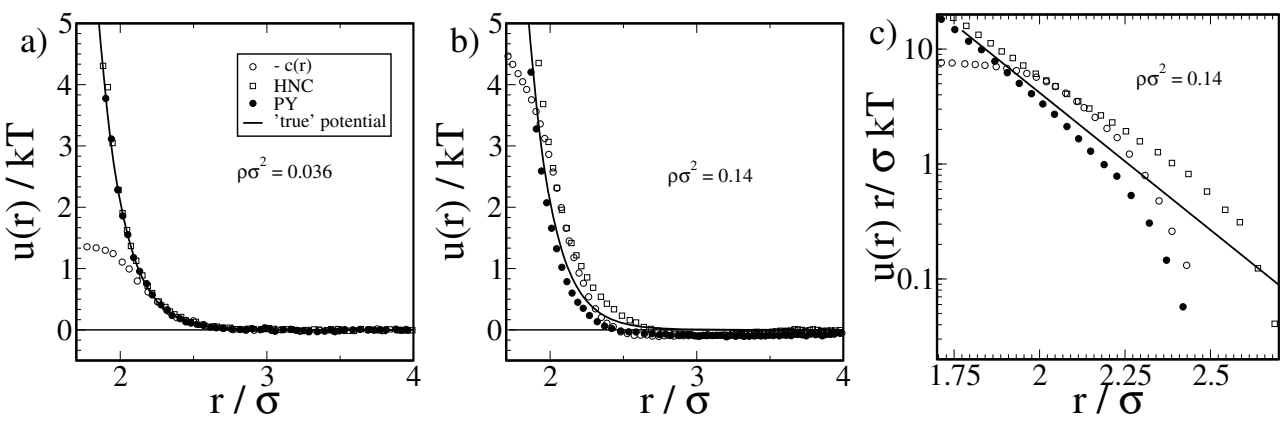

Figure 4. Pair potentials (symbols) extracted from the $g(r)$ of a simulated colloidal system, using the OZ equation and the HNC, the PY and the MSA CRs. The solid curve is for the true potential, used in the MC simulation. The inversion procedure is seen to produce reliable potentials at low density in (a), but slightly inaccurate potentials at higher densities in (b). (c) shows the same data as (b), but now $u^{\text {eff }}(r)$ is multiplied by $r$ and plotted logarithmically.
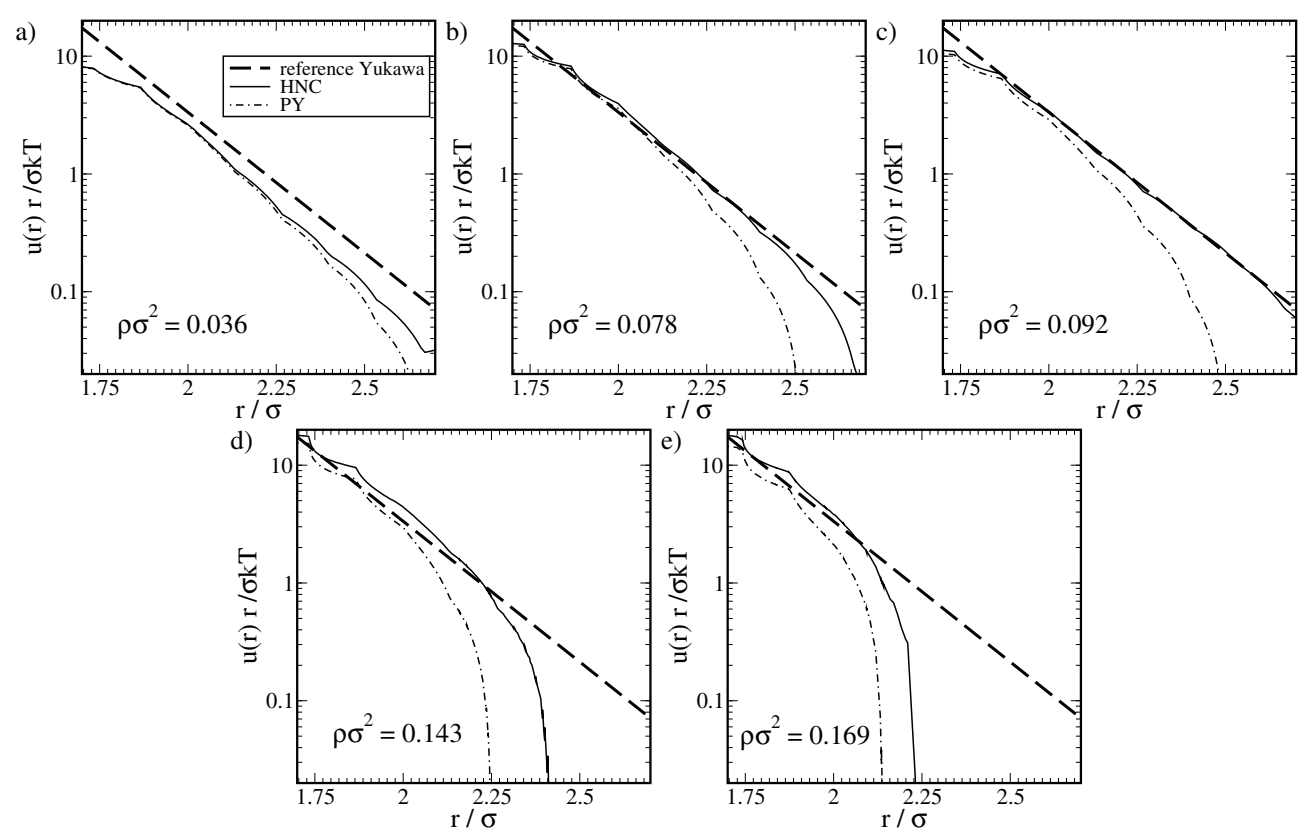

Figure 5. Effective colloid-colloid pair potentials $u^{\text {eff }}(r)$, obtained from inverting the measured $g(r)$ in figure 3. Potentials are multiplied by $r$ and plotted logarithmically, for different colloid densities as indicated. The thick dashed curve corresponds to the reference Yukawa potential, $\beta u^{\text {eff }}(r)=A \sigma \exp (-\kappa r) / r$ with $A=2.5 \times 10^{5}$ and $\kappa \sigma=5.5$.

standard MC simulations for a 2D Yukawa fluid at all six colloid densities investigated here and computed PDFs which are given by the solid lines in figure 3. For the three lowest densities the agreement between the measured and the simulated $g(r)$ in figure 3 is excellent.

For increasing density, however, the agreement between the experimental and numerical data in figure 3 becomes systematically poorer; the MC simulation using the Yukawa pair potential slightly underestimates the structure. This might at first glance be considered a negligible discrepancy, but it has significant consequences with respect to the effective 


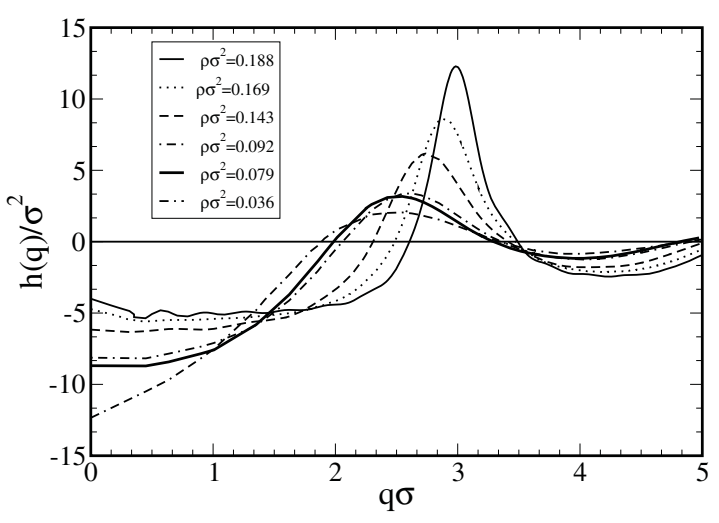

Figure 6. The Fourier transform of the total correlation function $h(r)=g(r)-1$ corresponding to the six measured radial distribution functions in figure 3.

potentials. Figure 4(c) shows the potentials obtained from inverting the simulated $g(r)$ curve for $\rho \sigma^{2}=0.143$ in figure 3 , and figure 5(d) the potentials from inverting the measured PDFs for $\rho \sigma^{2}=0.143$. We see that the $\mathrm{OZ}$ inversion procedure produces results that are consistent with the Yukawa potential in figure 4(c), but inconsistent with it in figure 5(d). Expecting the true potential to be located somewhere between the HNC-based and the PY-based potential, we recognize from figures 5(d) and (e) that $u^{\text {eff }}(r)$ is Yukawa-like only at very short distances and that it shows clear deviations from the Yukawa form at larger distances. Looking at the whole sequence of pictures in figure 5 , one can easily identify a density effect on the pair potential. A density dependence of the pair potential means only that the mutual interaction of two colloidal particles depends on the local environment that they are in. Two colloids isolated in an unbound electrolyte interact differently to two colloids at the same distance and at the same salinity but in a highly concentrated suspension. The interaction thus depends on other colloidal particles in the suspension. The density dependence of the pair potential recognizable in figure 5 is a direct experimental observation of many-body interactions in colloidal suspensions. Another observation known from the literature [30] evidences that elastic properties of colloidal crystals formed in charge-stabilized suspensions cannot be ascribed to the linear superposition of pairwise interactions, which is likely to be a similar consequence of many-body interactions.

We also observe from figure 5 that at those densities/distances where the potentials actually have Yukawa form, the values for the screening parameter $\kappa$, i.e. essentially the slope of the straight lines in figure 5, is independent of the colloid densities. This confirms our expectation that due to the construction of our cell we have realized a situation where the salinity is indeed fixed. This is important because otherwise a trivial density dependence of the pair potential would have occurred which would have masked the more interesting density effect coming from the many-body interaction. Note also the vertical shifts of the curves in figure 5; they indicate a weak density dependence of the prefactor of the effective pair potential which is consistent with a slight increase of the effective saturated charge with the colloid density, expected for such systems [16].

It is also instructive to study the Fourier transform $\hat{h}(q)$ of the total correlation function which is essentially the Fourier transform of the $g(r)$ plotted in figure 3. They are given in figure 6 for all six densities measured. One observes a systematic formation of a shallow minimum around $q \sigma \approx 1$. Approaching $q \sigma=0$, one would expect a monotonic decrease for a simple repulsive pair potential which is obviously not the case here. It is interesting to 


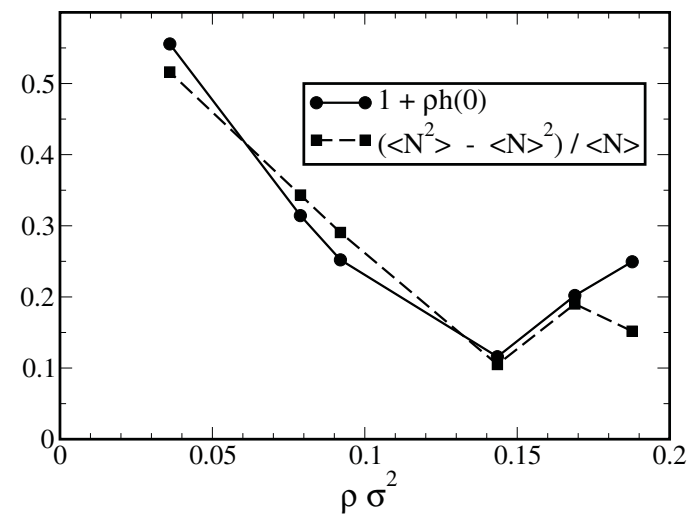

Figure 7. The values of $\hat{h}(q)$ at $q=0$ from figure 6, plotted as a function of the colloid density (filled circles), and the particle fluctuation in the system (filled squares).

inspect more closely the value at $\hat{h}(0)$ which we know from equation (15) to be related to the particle fluctuations $\Delta N$ in the system. This is done in figure 7. Our evaluation routine used to analyse the experimental data allows us not only to calculate $g(r)$ and thus $h(r)$, but also (and independently of the evaluation of $g(r)$ ) to determine the right-hand side of equation (15); the results are given as square symbols in figure 7. The two quantities should in principle be identical; the small difference in figure 7 can be attributed to the numerical error produced by the Fourier transformation. As expected, the relative fluctuation decreases with increasing density, but shows the interesting feature of fluctuation increasing again at higher densities.

\section{Results-inversion using the inverse Monte Carlo simulation method}

At $\rho \sigma^{2}=0.1689$, the potentials obtained using the $\mathrm{OZ}$ equation together with the HNC, PY and MSA CRs already show significant differences; at the largest density, $\rho \sigma^{2}=0.1877$, the OZ-based inversion routine can no longer produce physically meaningful effective potentials. We therefore apply another, more rigorous method of solving the inversion problem - the inverse Monte Carlo (IMC) method, due to Lyubartsev and Laaksonen [31]. The method is based on exact statistical-mechanical relations between the pair potential and the PDF which exist in case the system possesses pairwise-additive interactions only [29, 31]. The existence of these relations implies that two pair potentials producing the same PDF can differ from each other only by an additive constant. If one applies a natural constraint $u^{\text {eff }}(\infty)=0$, one obtains an unique solution of the inversion problem. The method combines solving these relations for a chosen reference PDF and testing the effective potential against the structure of the reference system using a MC simulation. The IMC technique was successfully applied to ionic solutions [32] and charged colloids [35].

The method consists of the following steps.

(i) Setting initial pair potentials between the particles of the one-component system. In most cases, the potential of mean force is a good first choice. If the pair potential for a system with similar characteristics is known, it can be taken instead. The potential is then presented in tabulated form on a mesh corresponding to the division of the original PDF, and the intermediate values are calculated using interpolation.

(ii) MC simulation of the OCM with the chosen potential; calculation of the PDF. 

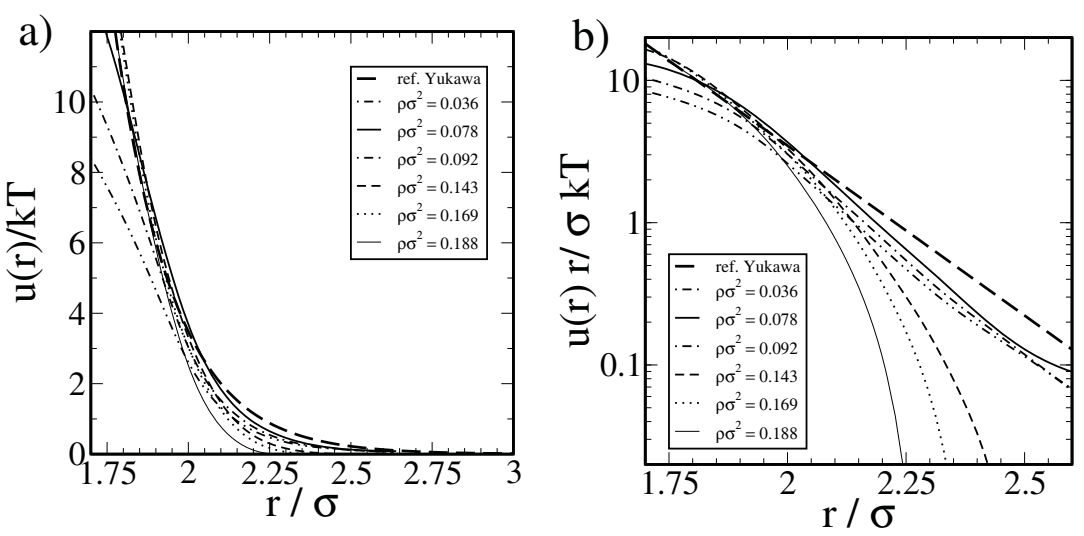

Figure 8. (a) The short-range part of effective macroion-macroion potentials for different densities obtained from the IMC procedure at the indicated colloid density obtained with cut-off of $r \approx 3 \sigma$. (b) The same curves as in (a), but $u^{\text {eff }}(r)$ is now multiplied by $r$ and plotted logarithmically.

(iii) Comparison of the PDF thus obtained with the reference PDF; solving a system of linear equations relating the differences in PDFs to the corrections for each potential bin.

(iv) Steps (ii), (iii) are repeated until convergence. Usually, no more than ten iterations are required.

Applying the IMC procedure to the data of figure 3, we find that the relative accuracy of calculation of $u^{\text {eff }}(r)$ strongly depends on the distance $r$. The essential measure here is the mean distance $D=\rho^{-1 / 2}$ between two colloids in the suspension. While the short-range part of the potential is stable at any conditions, the long-range part $(r>D)$ is seriously affected by the imposed cut-off. Note that the latter part expresses rather indirect interactions as the direct potential is already very small at the mean interparticle distance. We therefore compare only the short-range parts of the effective potentials. They are shown in figure 8(a) on a linear scale, and (multiplied by $r$ ) in figure 8(b) on a linear-log scale. The results are in a good agreement with the results of the previous section for the OZ-based inversion scheme. It is seen that in consistency with our observations from figure 5, there is a deviation from the Yukawa shape, which grows larger on increasing the colloid density.

To ensure universality of the mechanisms influencing the interactions at higher densities, we modelled this experiment by means of MC primitive model simulation. Direct simulation of a system of $3 \mu \mathrm{m}$ particles with the present charge density would have required a set-up with at least $10^{8}$ charged species involved (or $10^{6}$ for the effective charge) which is currently unfeasible. We therefore chose a system which allowed us to mimic the experimental one qualitatively. Specifically, we reproduced the surface charge density and the $\kappa \sigma$ value. To this end we simulated a 3D asymmetric electrolyte with macroions confined in two dimensions. Small ions were allowed to move freely in three dimensions and periodic boundary conditions in all three dimensions were applied. We can safely assume that the periodic images of the macroionic layer do not affect the interactions within the layer as we set the distance between the images to be at least three times the mean distance between the particles. To simulate the constant electrolyte strength conditions, a simple salt was added so that the total equilibrium electrolyte concentration (counterions + salt) in the macroion layer was always the same at all colloid densities.

Six different 2D particle number densities corresponding to the experimental ones were considered. The system contained 15 macroions of diameter $\sigma_{M}=40 \AA$ and charge 


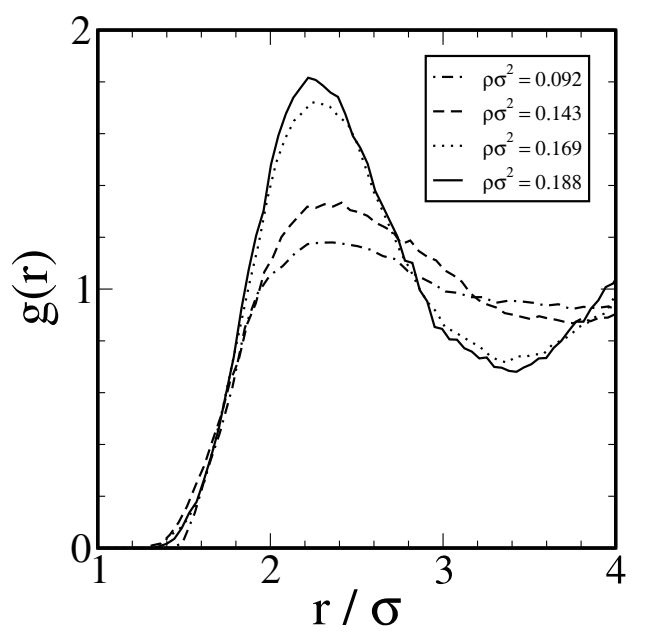

Figure 9. Colloid-colloid radial distribution functions for the model 2D colloidal suspension as obtained from primitive model MC simulation at different colloid densities.

$Z_{M}=-60,1500$ point-like monovalent counterions and added monovalent salt with pointlike anions and cations of $\sigma_{M}=5 \AA\left(T=298, \varepsilon_{r}=78.4\right)$. For the highest-density sample, no salt was added, while for the less dense ones, the salt concentration had to be adjusted. Thus, the salt concentration for the smallest density was about five times the ionic strength corresponding to counterions only. For equilibration, 5000 attempted MC steps per particle were carried out, while for the production runs we performed at least 50000 steps per particle. We calculated two-dimensional radial distribution functions for the macroions in the plane. The simulation package MOLSIM was used [33]. Periodic boundary conditions, Ewald summation for handling the long-range electrostatic interaction and the cluster move technique for improving the MC sampling were employed; further details of the simulation technique can be found in $[34,36]$.

The resulting PDFs for the four highest densities are presented in figure 9. The data for the two most diluted samples did not display distinguishable structure. We note qualitative agreement between the experimental set of curves and the data of the simulation. The first peaks of the PDFs are located at about the same position, 2.2 $\sigma$. The inversion procedure was applied as described above and its results are presented in figure 10. The effective pair potentials extracted from the simulation data appear similar to the potentials for the experimental suspension. They display Yukawa-like decay at separations between $1.7 \sigma$ and $3 \sigma$ and become negligibly small for $r>3 \sigma$. We note again that the curves for the two highest densities exhibit a downturn and decay to zero faster than those for the smaller densities, in agreement with the experimental results. The effect here is less pronounced than for the experimental system which can be attributed to the lower charges and weaker interactions in the MC system.

\section{Discussion and concluding remarks}

It has to be emphasized again that the inversion of the observed PDFs results in potentials which are to be distinguished from those appearing in the total potential of mean force in the McMillan-Mayer sense. Possibly containing also higher-order contributions, $u^{\text {eff }}(r)$ is of 


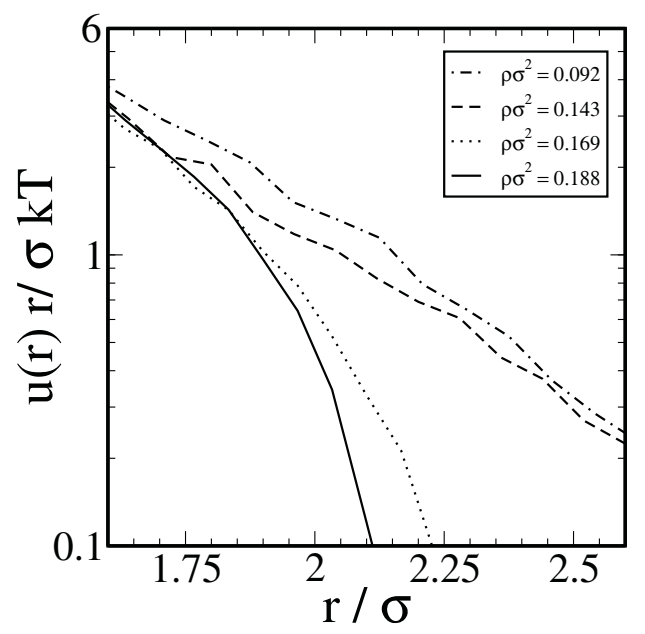

Figure 10. Effective potentials for the model 2D colloidal suspension as obtained from primitive model MC simulation and the IMC procedure at different colloid densities.

qualitatively different nature to the pair potential $v^{\text {eff }}(r)$. Whereas $v^{\text {eff }}(r)$ is of DLVO form, our main finding on the basis of two independent inversion procedures is a non-trivial density dependence of $u^{\text {eff }}(r)$, meaning that not only does $u^{\text {eff }}(r)$ differ from $v^{\text {eff }}(r)$ by having different density-dependent parameters, but also the functional form differs. It is Yukawa-like, as in DLVO theory, at low densities, but shows clear deviations from this form at higher densities.

A qualitative understanding of our results follows from the observation that deviations of the measured potentials in figures 5 and $8(\mathrm{~b})$ take place at distances comparable to the mean distance $D=\rho^{-1 / 2}(D / \sigma=2.3,2.4,2.6$ for the three highest densities and $D / \sigma=3.3,3.5,5.3$ for the three lowest colloid densities). Two colloids in a suspension which are separated by $r>D$ are likely to have a third particle somewhere in between. By contrast, the potential in equation (8) is based on the assumption of two (and just two) interacting colloids, each surrounded by an unperturbed spherical double layer. This clearly cannot give the correct interaction potentials in cases where a third macroion in between is effectively blocking the mutual interaction of the two colloids. Following this interpretation, the deviation of the functional form of $u^{\mathrm{eff}}(r)$ from that of the Yukawa potential arises from $N$-body $(N \geqslant 3)$ potentials of the McMillan-Mayer expansion in equation (7), absorbed into $u^{\text {eff }}(r)$. We recall from the discussion of three-particle interactions at the end of section 2 that three-body potentials in our systems are attractive, and that they nearly cancel the repulsive pair potential between macroions when another one lies in between. This latter condition is usually satisfied when $r>D$, and it is just in this distance regime that we find the deviations of $u^{\text {eff }}(r)$ from DLVO form. It seems therefore appropriate to call this effect 'macroion screening' Taken altogether, our experiment represents a direct observation of many-body interactions in colloidal suspensions.

Macroion screening is not important in suspensions at low colloid density, since in these systems the pair potential has decayed essentially to zero for $r \geqslant D$ due to microion screening, so the shielding effect of macroions cannot be observed. It is important to note that qualitatively similar deviations of the effective pair potential from the Yukawa form are found also in the primitive model calculations reported in section 5, although the simulations could not be performed for parameters exactly corresponding to the experiment. The resulting effective 
pair potentials $u^{\text {eff }}(r)$ show the very same features as those obtained from the experiments. The observed deviations are such that the effective potentials become very small for $r \geqslant D$ which is interpreted as a cancellation of the DLVO potential by effective three-body potentials.

We finally comment on the existence of minima at large distances, which we found in $u^{\text {eff }}(r)$ with both inversion routines used. Using the OZ-based inversion, we observe a very shallow minimum of $0.2 k T$ at $\rho \sigma^{2}=0.1438$ and $0.5 k T$ at $\rho \sigma^{2}=0.1689$ at larger distances which however were already of the same order as the differences between the HNC-based and the PY-based potentials. Looking back to figure 4, one also recognizes a shallow minimum which here is clearly an artefact of the inversion procedure. From that, one must conclude that the results of the inversion based on the $\mathrm{OZ}$ equation, at higher densities and larger distances have to be interpreted with utmost caution. However, a shallow minimum in $u^{\text {eff }}(r)$ has also been found using the IMC inversion procedure (not visible in figure 8(a)). The minimum is located at about $r=2.7 \sigma$ (the PDF peak position) for the three lowest concentrations and is systematically displaced towards smaller separation for increasing densities. The depth of the minimum varies from $0.1 k T$ at the lower densities to about $k T$ at the highest one at a fixed interaction cut-off distance. At the same time, the depth is very sensitive to the chosen cut-off and decreases with shortening cut-off at a fixed density.

Other authors who used a similar OZ-based inversion procedure also reported on attractive forces in charged colloidal suspensions [23, 24, 27, 28], and interpreted them as evidence for 'like-charge attraction'. Because most of these experiments were performed in very thin 2-6 $\mu \mathrm{m}$ glass cells, it has been speculated that the origin of the attraction may arise from electrostatic influences of the confining glass plates. Owing to the fact that the observed pair potentials show also attractive potential wells of a few tenths of $k_{B} T$ depth at similar distances, however, we suggest explaining their observations also in terms of macroion screening effects as discussed above. This is also supported by a very similar density dependence found in [26] and [28] which clearly indicates that the observed attraction is a many-body effect.

In conclusion, we have performed density-dependent measurements of the effective pair potentials in charge-stabilized 2D colloidal suspensions. Deviations from a Yukawa potential were observed at higher densities which we interpreted in terms of many-body interactions. This interpretation is also consistent with other experimental studies observing attractive parts in the colloid/colloid pair potential which were interpreted as 'like-charge attraction'. Our study demonstrates that $v^{\text {eff }}(r)$ at these conditions is always repulsive and 'like-charge attraction', or the appearance of a minimum in $u^{\mathrm{eff}}(r)$, might in fact be nothing but a minimum caused by absorbing many-body interactions into the pair potential.

\section{Acknowledgments}

We gratefully acknowledge the financial support from the DFG through SFB 513. We also thank Alexander Lyubartsev for giving us access to his IMC code and Wolfram Strepp for providing the MC simulation data [11] in figure 3.

\section{References}

[1] Nägele G 1996 Phys. Rep. 272215

[2] Löwen H and Hansen J-P 2000 Annu. Rev. Phys. Chem. 51209

[3] Vlachy V 1999 Annu. Rev. Phys. Chem. 50145

[4] Pusey P N 1991 Liquids, Freezing and Glass Transition part II ed J-P Hansen, D Levesque and J Zinn-Justin (Amsterdam: Elsevier) p 763

[5] Ise N, Konishi T and Yamanaka J 2001 Curr. Opin. Colloid Interface Sci. 6126

[6] Belloni L 2000 J. Phys.: Condens. Matter 12 R549 
[7] Verwey E J W and Overbeek J T G 1948 Theory of the Stability of Lyophobic Colloids (Amsterdam: Elsevier)

[8] Evans D F and Wennerström H 1994 The Colloidal Domain: Where Physics, Chemistry, Biology, and Technology Meet (New York: VCH)

[9] Attard P 2001 Curr. Opin. Colloid Interface Sci. 6366

[10] Arora A K and Tata B V R 1998 Adv. Colloid Interface Sci. 7849

[11] Brunner M, Bechinger C, Strepp W, Lobaskin V and von Grünberg H H 2002 Europhys. Lett. 58926

[12] Bell G M, Levine S and McCartney L N 1970 J. Colloid Interface Sci. 33335

[13] Deserno M and von Grünberg H H 2002 Phys. Rev. E 66011401

[14] Beresford-Smith B, Chan D Y C and Mitchell D J 1985 J. Colloid Interface Sci. 2169691

[15] Chan 2001 Phys. Rev. E 63061806

Chan D Y C, Linse P and Petris S N 2001 Langmuir 174202

[16] Belloni L 1998 Colloids Surf. A 140227

[17] Löwen H and Allahyarov E 1998 J. Phys.: Condens. Matter 104147

Wu J Z, Bratko D, Blanch H W and Prausnitz J M 2000 J. Chem. Phys. 1133360

[18] Denton A R 1999 J. Phys.: Condens. Matter 11 10061-71

[19] Russ C, van Roij R, Dijkstra M and von Grünberg H H 2002 Phys. Rev. E 66011402

[20] Palberg T, Härtl W, Wittig U, Versmold H, Würth M and Simnacher E 1992 J. Phys. Chem. 968180

[21] Helden L, Leiderer P and Bechinger C 2001 unpublished data

[22] Burns M M, Fournier J-M and Golovchenko J A 1989 Phys. Rev. Lett. 631233

[23] Cruz de León G and Arauz-Lara J L 1999 Phys. Rev. E 594203

[24] Quesada-Perez M, Moncho-Jorda A, Martinez-Lopez F and Hidalgo-Alvarez R 2001 J. Chem. Phys. 11510897

[25] Faucheux L P, Stolovitzky G and Libchaber A 1995 Phys. Rev. E 515239

[26] Kepler G M and Fraden S 1994 Phys. Rev. Lett. 73356

[27] Cruz de León G, Saucedo-Solorio J M and Arauz-Lara J L 1998 Phys. Rev. Lett. 811122

[28] Carbajal-Tinoco M D, Castro-Román F and Arauz-Lara J L 1996 Phys. Rev. E 533745

[29] Henderson R L 1974 Phys. Lett. A 49197

[30] Weiss J A, Larsen A E and Grier D G 1998 J. Chem. Phys. 1098659

[31] Lyubartsev A P and Laaksonen A 1995 Phys. Rev. E 523730

[32] Lyubartsev A P and Laaksonen A 1997 Phys. Rev. E 555689

[33] We used the simulation package MOLSIM (MOLSIM, V 3.2, P Linse, Lund University, 2002).

[34] Lobaskin V and Linse P 1999 J. Chem. Phys. 1114300

[35] Lobaskin V, Lyubartsev A and Linse P 2001 Phys. Rev. E 111020401

[36] Linse P and Lobaskin V 2000 Simulation and Visualization on the Grid (Lecture Notes in Computational Science and Engineering Series vol 13) ed B Engquist, L Johnsson, M Hammill and F Short (Berlin: Springer) p 165 